\title{
Studies on Orchidaceae Glycosides. 2.* The Structures of Loroglossine and Militarine, two Glucosides from
}

\section{Orchis militaris L.}

\author{
ARNE AASEN, ${ }^{a}$ DAN BEHR $^{b}$ and KURT LEANDER ${ }^{b}$
}

\begin{abstract}
a Research Department, Swedish Tobacco Co., Box 17007, S-104 62 Stockholm, Sweden and b Department of Organic Chemistry, Arrhenius Laboratory, University of Stockholm, S-104 05 Stockholm, Sweden
\end{abstract}

Two glucosides, loroglossine (I) and militarine (II), have been isolated from Orchis militaris L. Loroglossine (I) is shown to be bis[4-( $\beta$-D. glucopyranosyloxy)benzyl] erythro-isobutyltartrate and militarine (II) bis[4-( $\beta$-D-glucopyranosyloxy)benzyl] (R)-2-isobutylmalate. Syntheses of the $( \pm)$-erythro and $( \pm)$-threo isomers of dimethyl isobutyltartrate are described.

In 1919 Bourquelot and Bridel ${ }^{2}$ isolated a glucoside from Loroglossum hircinum (L.) L.C. Rich. for which they proposed the name loroglossine. This glucoside was later found in Orchis militaris L. and twenty-one other Orchidaceae species. ${ }^{3-7}$

In this communication we report the structure of loroglossine (I) and another glucoside, for which we propose the name militarine (II), both of which were isolated from $O$. militaris.

The two glucosides, which differ by only one hydroxyl group, have the molecular weights 742 and 726, respectively. In spite of the small differences in size and structure, I and II were separated by gel permeation on Sephadex G-15.

Loroglossine (I). On the basis of elemental analysis and molecular weight determinations, Delauney $^{8}$ suggested the molecular formula $\mathrm{C}_{30} \mathrm{H}_{42} \mathrm{O}_{18}$ for loroglossine (I). Later Karrer and Matter ${ }^{\circ}$ prepared some derivatives of I and from the results of the elemental analysis of these, they considered loroglossine (I) to be a mono-glucoside with the composition $\mathrm{C}_{14} \mathrm{H}_{20} \mathrm{O}_{8}$.

\footnotetext{
* For paper 1 in this series, see Ref. 1 .
}

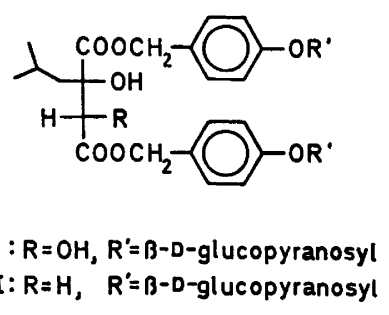

In the present study, spectrochemical and elemental analyses indicated that loroglossine (I) has the molecular formula $\mathrm{C}_{34} \mathrm{H}_{48} \mathrm{O}_{18}$. Sugar and methylation analyses ${ }^{10}$ showed $\mathrm{I}$ to be a glucopyranoside. Catalytic hydrogenation of I produced $p$-cresyl $\beta$-D-glucopyranoside ${ }^{11}$ and isobutyltartaric acid. As there are no signals due to aromatic methyl groups present in the NMR spectrum of I, loroglossine (I) must have suffered hydrogenolysis during the reduction. This evidence together with the NMR spectrum of I, which shows inter alia eight aromatic protons, indicates the presence of two 4-( $\beta$-Dglucopyranosyloxy)benzyl residues in loroglossine (I). Since loroglossine (I) is a neutral compound it must be a diester and hence have the structure $I$.

The relative configuration of the isobutyltartaric acid was established by synthesis. Hydroxylation of isobutylfumaric acid ${ }^{12}$ and isobutylmaleic anhydride ${ }^{12}$ with osmium tetroxide gave, after methylation $( \pm)$-threo and ( \pm )-erythro dimethyl isobutyltartrate, respectively. The erythro isomer was found to be identical, except for the optical rotation, with the dimethyl ester of the natural acid.

Acta Chem. Scand. B 29 (1975) No. 10 
Attempts to determine the absolute configuration of the isobutyltartaric acid are in progress.

Militarine (II). The molecular formula for militarine (II) was found to be $\mathrm{C}_{34} \mathrm{H}_{46} \mathrm{O}_{17}$, i.e. one oxygen atom less than loroglossine (I). Catalytic hydrogenation of II gave $p$-cresyl $\beta$-D-glucopyranoside ${ }^{11}$ and $(R)$-2-isobutylmalic acid.13,14 From the spectral similarities of loroglossine (I) and militarine, it is evident that the latter has the structure II.

$p$-Hydroxybenzyl alcohol has been isolated from $O$. militaris infected by the fungus Rhizoctonia repens Bern..$^{16-17}$ Since this alcohol is normally absent from the plant, ${ }^{15-17}$ it seems probable that it has been released from loroglossine (I) and/or militarine (II) by enzymatic hydrolysis during the period of infection.

\section{EXPERIMENTAL}

All melting points are corrected. Mass spectra were measured on a Perkin-Elmer 270 or a Varian MAT 311 spectrometer, optical rotations on a Perkin-Elmer 141 polarimeter, IR spectra on a Perkin-Elmer 257 instrument, UV spectra on a Beckman DK2 instrument, and NMR spectra on a Varian XL 100 spectrometer. Concentrations were performed under diminished pressure, at bath temperatures not exceeding $40^{\circ} \mathrm{C}$. Elemental analyses were carried out at Alfred Bernhardt, Mikroanalytisches Laboratorium, Elbach über Engelskirchen, Germany and Mikroanalyslaboratoriet Lantbrukshögskolan, Uppsala, Sweden.

Isolation and characterisation of $I$ and $I I$. Fresh plants of $O$. militaris $(5.5 \mathrm{~kg})$ were extracted with methanol (15 l). The filtrate was concentrated to $0.7 \mathrm{l}$, washed with chloroform $(3 \times 0.251)$ and extracted with butanol $(13 \times 0.15$ 1). The butanolic phase was washed with water $(0.15 \mathrm{l})$ and evaporated to dryness. Half of the residue (14 g) was filtered through a column of Sephadex LH $20(8 \times 60 \mathrm{~cm})$ with waterethanol $(1: 1,1 \mathrm{ml} / \mathrm{min})$ as eluent. The fraction $(4.6 \mathrm{~g})$ containing the glucosides was chromatographed on the same column giving a mixture of I and II (3.4 g). Crystallisation from water gave I $(0.9 \mathrm{~g})$ as needles, m.p. $153-157^{\circ} \mathrm{C}$ (decomp). $[\alpha]_{\mathrm{D}}{ }^{25}-34^{\circ}$ (c 0.36 , methanol). (Lit. ${ }^{8}$ m.p. $149-150^{\circ} \mathrm{C},[\alpha]_{\mathrm{D}}-36.28^{\circ}$, methanol. Lit. ${ }^{\circ}$ m.p. $149-151^{\circ} \mathrm{C}$ ). (Found: C 55.1; H 6.36; O 38.7. Calc. for $\mathrm{C}_{34} \mathrm{H}_{48} \mathrm{O}_{18}$ : C 55.0; $\mathrm{H}$ 6.24; O 38.8). IR: $v_{\max }(\mathrm{KBr}) 3600-3000(\mathrm{~s})$, $1735(\mathrm{~s}), 1615(\mathrm{~m}), 1515(\mathrm{~s}) \mathrm{cm}^{-1}$. NMR (pyridine$\left.d_{5}\right): \delta 0.85(\mathrm{~d}, 3 \mathrm{H}, J 6 \mathrm{~Hz}), 0.97(\mathrm{~d}, 3 \mathrm{H}, J 6$ $\mathrm{Hz}), 1.8-2.2(\mathrm{~m}, 2 \mathrm{H}), 2.2-2.5(\mathrm{~m}, \mathrm{l} \mathrm{H}), 3.8-$ $4.5(\mathrm{~m}, 12 \mathrm{H}), 4.98-5.16(\mathrm{~m}, 4 \mathrm{H}), 5.21(\mathrm{~m}, 2$ $\mathrm{H}), 5.6-6.8$ (broad $\mathrm{s}, 10 \mathrm{H}), 7.25(\mathrm{~m}, 8 \mathrm{H})$.
UV, $\mathrm{nm}(\varepsilon): \lambda_{\max }$ (methanol) 277 (1340), $270.5(1590), 223.5(25400)$.

Part of the mother liquor $(100 \mathrm{mg})$ from the crystallisation of I was chromatographed on Sephadex G $15(1.6 \times 70 \mathrm{~cm})$ with water as eluent, giving I (50 mg) and a mixture of $I$ and II $(20 \mathrm{mg})$. Further chromatography in the same system, followed by evaporation gave pure II as a colourless amorphous solid, $[\alpha]_{578}{ }^{25}$ $-47^{\circ}$ (c 0.78, methanol). (Found: C 56.2; $\mathrm{H}$ $6.60 ; \mathrm{O} 37.4$. Calc. for $\mathrm{C}_{34} \mathrm{H}_{46} \mathrm{O}_{17}$ : $\mathrm{C} 56.2 ; \mathrm{H}$ 6.38; O 37.4). IR: $v_{\max }(\mathrm{KBr}) 3700-3000(\mathrm{~s})$, $1735(\mathrm{~s}), 1615(\mathrm{~m}), 1515(\mathrm{~s}) \mathrm{cm}^{-1}$. NMR (pyridine. $d_{5}: \delta 0.90($ d. $3 \mathrm{H}, J 6 \mathrm{~Hz}), 0.97(\mathrm{~d}, 3 \mathrm{H}, J 6 \mathrm{~Hz}$ ), $1.7-1.94(\mathrm{~m}, 2 \mathrm{H}), 1.94-2.3(\mathrm{~m}, 1 \mathrm{H}), 2.99$ $(\mathrm{d}, 1 \mathrm{H}, J 15 \mathrm{~Hz})$ and $3.19(\mathrm{~d}, 1 \mathrm{H}, J 15 \mathrm{~Hz}$, $\mathrm{AB}$ system), $3.9-4.6(\mathrm{~m}, 12 \mathrm{H}), 5.08$ (s. $2 \mathrm{H})$, $5.17(\mathrm{~s}, 2 \mathrm{H}), 5.38-5.64(\mathrm{~m}, 2 \mathrm{H}), 5.8-7.2$ (broad s, $9 \mathrm{H}), 7.22-7.45$ (m, $8 \mathrm{H})$. UV, $\mathrm{nm}$ $(\varepsilon): \lambda_{\max }$ (methanol) 277 (1430), 270.5 (1730), $223(24900)$.

Hydrogenation of $I$ and $I I$. A solution of I $(140 \mathrm{mg})$ in methanol $(10 \mathrm{ml})$ was hydroge. nated over palladium ( $20 \mathrm{mg}, 10 \%$ on carbon) at room temperature and atmospheric pressure. After $2.5 \mathrm{~h}$ the catalyst was filtered off and the solution was evaporated to dryness. The residue was purified by preparative TLC on silica gel using chloroform-methanol (1:1) as eluent. Crystallisation from a mixture of propan-2-ol and water (3:1) gave $p$-cresyl $\beta$-D-glucoside (67 mg) m.p. $178-181^{\circ} \mathrm{C}$ (Lit.11 m.p. $178-179.5$ $\left.{ }^{\circ} \mathrm{C}\right),[\alpha]_{\mathrm{D}}{ }^{23}-66.5^{\circ}$ (c 2.26, water) (Lit. ${ }^{11}[\alpha]_{\mathrm{D}}{ }^{20}$ -67.7 ${ }^{\circ}$, water). IR: $v_{\max }(\mathrm{KBr}) 3490(\mathrm{~s}), 3420(\mathrm{~s})$, $3380(\mathrm{~s}), 1610(\mathrm{~m}), 1510(\mathrm{~s}) \mathrm{cm}^{-1}$. NMR (pyridine$\left.d_{5}\right): \delta 2.14(\mathrm{~s}, 3 \mathrm{H}), 3.9-4.6(\mathrm{~m}, 6 \mathrm{H}), 5.4-5.6$ $(\mathrm{m}, 1 \mathrm{H}), 6.6-7.3(\mathrm{~m}, 8 \mathrm{H})$. UV, $\mathrm{nm}(\varepsilon): \lambda_{\max }$ (methanol) 281 (1090), 275 (1370), 220 (11 200). The same compound (m.p., specific rotation, IR, NMR, and UV) was obtained when II was hydrogenated under the same conditions.

Dimethyl isobutyltartrate. I $(620 \mathrm{mg})$ was hydrogenated as described above. Evapora. tion of the solvent gave a residue which was purified by preparative TLC on silica gel using methanol-butanol-chloroform (1:3:6) as eluent. The eluate from the plates was filtered through Dowex $50 \mathrm{~W}-\mathrm{X} 8\left(\mathrm{H}^{+}, 1 \times 5 \mathrm{~cm}\right)$ using water as eluent. The filtrate was evaporated to dryness giving isobutyltartaric acid, which was esterified with diazomethane. The crude ester was purified by sublimation $\left(0.1\right.$ Torr, $\left.45^{\circ} \mathrm{C}\right)$ giving dimethyl isobutyltartrate $(67 \mathrm{mg})$ as white needles, m.p. $57-59^{\circ} \mathrm{C},[\alpha]_{578}{ }^{25}+31^{\circ}$ (c 0.43 , methanol). (Found: C 51.4; $\mathrm{H}$ 7.88; O 41.1. Calc. for $\mathrm{C}_{10} \mathrm{H}_{18} \mathrm{O}_{6}$ : C 51.3; $\mathrm{H}$ 7.75; $\mathrm{O} 41.0$.) IR: $v_{\max }\left(\mathrm{CHCl}_{3}\right) 3530(\mathrm{~m}), 1740(\mathrm{~s}) \mathrm{cm}^{-1}$. NMR $\left(\mathrm{CDCl}_{3}+\mathrm{D}_{2} \mathrm{O}\right): \delta 0.85(\mathrm{~d}, 3 \mathrm{H}, J$ 6.5 Hz) 0.95 $(d, 3 \mathrm{H}, J$ 6.5 Hz) $1.33-2.14(\mathrm{~m}, 3 \mathrm{H}), 3.73$ (s, $3 \mathrm{H}), 3.80(\mathrm{~s}, 3 \mathrm{H}), 4.33$ (s, $1 \mathrm{H}) . \mathrm{MS}, m / e$ (rel. intensity): $M^{+} 234$ (absent), 175 (7), 157 (4), 145 (4), 129 (4), 115 (7), 103 (5), 101 (18), 90 (100), $85(38), 73(9), 59(10), 57(42), 43(33)$.

Dimethyl (R)-2-isobutylmalate. II (150 mg) was hydrogenated as described above for I. 
The catalyst was filtered off and an excess of diazomethane in ether was added. After $1 \mathrm{~h}$, the reaction mixture was evaporated to dryness and the residue was filtered through a column of silica gel $(2.5 \times 7 \mathrm{~cm})$ using chloroform. methanol (9:1) as eluent. The crude dimethyl ester was purified by preparative GLC (5\% SE-52 on Chromosorb W, $3 \mathrm{~mm} \times 1.8 \mathrm{~m}, 130$ ${ }^{\circ} \mathrm{C}$, flow rate $40 \mathrm{ml} / \mathrm{min}$, retention time $4 \mathrm{~min}$ ) giving dimethyl ( $R$ )-2-isobutylmalate $(20 \mathrm{mg})$ as an oil $[\alpha]_{578}{ }^{25}-0.55^{\circ},[\alpha]_{436}{ }^{25}+0.30^{\circ},[\alpha]_{365}{ }^{25}$ $+5.3^{\circ}$ (c 2.0, othanol) [Lit. ${ }^{13,14}[\alpha]_{578}{ }^{24}-0.64^{\circ}$, $[\alpha]_{438}{ }^{24}+0.16^{\circ},[\alpha]_{385}{ }^{24}+3.9^{\circ}$ (c 1.0, ethanol) $]$. IR: $v_{\max }\left(\mathrm{CHCl}_{3}\right) 1740 \mathrm{~cm}^{-1}$. NMR $\left(\mathrm{CDCl}_{3}\right)$ : $\delta 0.85$ (d, $3 \mathrm{H}, J 6 \mathrm{~Hz}), 0.95$ (d, $3 \mathrm{H}, J 6 \mathrm{~Hz}$ ), $1.55-1.75(\mathrm{~m}, 3 \mathrm{H}), 2.73(\mathrm{~d}, 1 \mathrm{H}, J \mathrm{I} 6 \mathrm{~Hz})$ and 2.89 (d, $1 \mathrm{H}, J 16 \mathrm{~Hz}, \mathrm{AB}$ spectrum), 3.67 $(\mathrm{s}, 3 \mathrm{H})$; $3.79(\mathrm{~s}, 3 \mathrm{H}), 3.2-4.0$ (1 H, exchangeable in $\mathrm{D}_{2} \mathrm{O}$ ).

Dimethyl ( \pm )-erythro-isobutyltartrate. Isobutylmaleic anhydride ${ }^{12}$ (191 $\left.\mathrm{mg}\right)$ was dissolved in pyridine $(15 \mathrm{ml})$ and osmium tetroxide $(333$ $\mathrm{mg}$ ) was added. The mixture was stirred at room temperature for $\mathbf{3 5} \mathrm{min}$ after which sodium hydrogen sulfite $(0.6 \mathrm{~g})$, pyridine $(7 \mathrm{ml})$, and water $(10 \mathrm{ml})$ were added. After $30 \mathrm{~min}$ at room temperature the solution was heated to $60^{\circ} \mathrm{C}$ for $15 \mathrm{~min}$. The reaction mixture was evaporated to dryness and the residue was filtered through Dowex $50 \mathrm{~W}-\mathrm{X8}\left(\mathrm{H}^{+}, 2.5 \times 8\right.$ cm) using water as eluent. The dark eluate $(180 \mathrm{ml})$ was extracted with ethyl acetate $(6 \times 50$ $\mathrm{ml}$ ) and the organic phase was dried and evap. orated to dryness. The residue $(175 \mathrm{mg})$ was treated with diazomethane giving the crude ester which was purified by chromatography on silica gel $(2.5 \times 5.5 \mathrm{~cm})$ using chloroform as eluent. The fraction containing the dimethyl ester was purified by sublimation (0.02 Torr, $\left.45^{\circ} \mathrm{C}\right)$ giving dimethyl $( \pm)$-erythro-isobutyltartrate (114 mg), m.p. $80-82^{\circ} \mathrm{C}$, indistinguishable (IR, NMR, MS, and GLC) from the natural product.

Dimethyl ( \pm )-threo-isobutyltartrate. Isobutylfumaric acid ${ }^{12}(220 \mathrm{mg})$ was reacted as described above for isobutylmaleic anhydride, except that the heating was omitted. Sublimation $\left(0.02\right.$ Torr, $\left.50^{\circ} \mathrm{C}\right)$ gave dimethyl $( \pm)$-threoisobutyltartrate $(112 \mathrm{mg})$ m.p. $50-52^{\circ} \mathrm{C}$. IR: $v_{\max }\left(\mathrm{CHCl}_{\mathrm{s}}\right) 3530(\mathrm{~m}), 1740(\mathrm{~s}) \mathrm{cm}^{-1}$. NMR $\left(\mathrm{CDCl}_{3}\right): \delta 0.83(\mathrm{~d}, 3 \mathrm{H}, J 6 \mathrm{~Hz}), 0.94(\mathrm{~d}, 3 \mathrm{H}$, $J 6 \mathrm{~Hz}), 1.63-1.92(\mathrm{~m}, 3 \mathrm{H}), 3.83(\mathrm{~s}, 6 \mathrm{H})$, $4.28(\mathrm{~s}, 1 \mathrm{H})$.

Acknowledgements. We are indebted to Dr. Björn Lüning for supplying the plant material and for his interest in this work. We thank the Swedish Natural Science Research Council for support.

\section{REFERENCES}

1. Behr, D., Berg, J.-E., Karlsson, B., Leander, K., Pilotti, A.-M. and Wiehager, A.-C. Acta Chem. Scand. B 29 (1975) 401.
2. Bourquelot, E. and Bridel, M. C. R. Acad. Sci. 168 (1919) 701.

3. Delauney, P. C. R. Acad. Sci. 171 (1920) 435.

4. Delauney, P. J. Pharm. Chim. 23 (1921) 265.

5. Delauney, P. C. R. Acad. Sci. 176 (1923) 598.

6. Delauney, P. C. R. Acad. Sci. Ser. C. 180 (1925) 224.

7. Charaux, C. and Delauney, P. C. R. Acad. Sci. 180 (1925) 1770.

8. Delauney, P. Contribution a l'étude des glucosides de la famille des Orchidées, Diss., Univ. Paris 1924.

9. Karrer, P. and Matter, E. Helv. Chim. Acta 30 (1947) 2096.

10. Björndal, H., Hellerqvist, C. G., Lindberg, B. and Svensson, S. Angew. Chem. Int. Ed. Engl. 9 (1970) 610.

11. Semke, L. K., Thompson, N. S. and Williams, D. G. J. Org. Chem. 29 (1964) 1041. 12. Vaughan, W. R. and Andersen, K. S. J. Amer. Chem. Soc. 77 (1955) 6702.

13. Brandänge, S., Lüning, B., Moberg, C. and Sjöstrand, E. Acta Chem. Scand. 25 (1971) 349.

14. Brandänge, S., Josephson, S. and Vallén, S. Acta Chem. Scand. B 28 (1974) 153.

15. Gäumann, E. and Kern, H. Phytopathol. $Z 35$ (1959) 347.

16. Gäumann, E. and Kern, H. Phytopathol. Z. 36 (1959) 1 .

17. Hardegger, E., Schellenbaum, M. and Corrodi, H. Helv. Chim. Acta 46 (1963) 1171.

Received April 9, 1975. 